\title{
Monolithic Folded Pendulum Accelerometers for Seismic Monitoring and Active Isolation Systems
}

\author{
Alessandro Bertolini, Riccardo DeSalvo, Francesco Fidecaro, and Akiteru Takamori
}

\begin{abstract}
A new class of very low noise low-frequency forcebalance accelerometers is presented. The device has been designed for advanced mirror isolation systems of interferometric gravitational wave detectors. The accelerometer consists of a small monolithic folded pendulum with $2 \mathrm{~s}$ of natural period and an in-vacuum mechanical quality factor of $\mathbf{3 0 0 0}$. The folded pendulum geometry, combined with the monolithic design, allows a unique $0.01 \%$ cross-axis residual coupling. Equipped with a high-resolution capacitance position sensor, it is capable of a noise-equivalent inertial displacement of 1-nm root mean square integrated over all the frequencies above $0.01 \mathrm{~Hz}$. The main features of this new accelerometer are here reviewed. New possible applications of monolithic folded pendula in geophysical instrumentation are discussed.
\end{abstract}

Index Terms-Accelerometer, pendulum, tiltmeter.

\section{INTRODUCTION}

A CTIVE seismic isolators for interferometric gravitational wave detectors (IGWD) rely on low-noise low-frequency accelerometers. Suppression of the off-band mirror residual motion is supported by means of either external active preisolation, as HEPI in present LIGO, and in-vacuum active damping of the internal resonances of the isolators, as in VIRGO Super-attenuators [1], TAMA-SAS [2] and in the advanced LIGO configurations [3]. While off-the-shelf seismometers perfectly match sensitivity and operation requirements of external active preisolators, in-vacuum devices must concern with more stringent constraints. These accelerometers must enable the hierarchical mirror control system to deliver an off-band integrated root mean square (rms) residual speed below $1 \mu \mathrm{m} / \mathrm{s}$ for easy lock acquisition, preserving a reasonable signal-to-noise ratio (SNR) over the control system frequency band. A noise-equivalent inertial displacement between 1-10-nm rms integrated over all the frequencies above $0.01 \mathrm{~Hz}$ is desired. A low cross-axis sensitivity is also required for operation in multidegree of freedom feedback. Moreover, they must be working in a high-vacuum environment with no maintenance or failure allowable on the timescale (years) of the interferometer data runs. State-of-the-art seismometers

Manuscript received April 12, 2005; revised August 26, 2005. This work was supported in part by the U.S. National Science Foundation under Grant LIGO PHY-0107417.

A. Bertolini was with Dipartimento di Fisica, Università di Pisa, 56127 Pisa, Italy. He is now with Deutsches Elektronen-Synchrotron (DESY), 22607 Hamburg, Germany (e-mail: alessandro.bertolini@ desy.de).

R. DeSalvo is with California Institute of Technology, Pasadena, CA 91125 USA.

F. Fidecaro is with Dipartimento di Fisica, Università di Pisa, 56127 Pisa, Italy.

A. Takamori is with the Earthquake Research Institute, University of Tokyo, 113-0033 Tokyo, Japan.

Digital Object Identifier 10.1109/TGRS.2005.861006

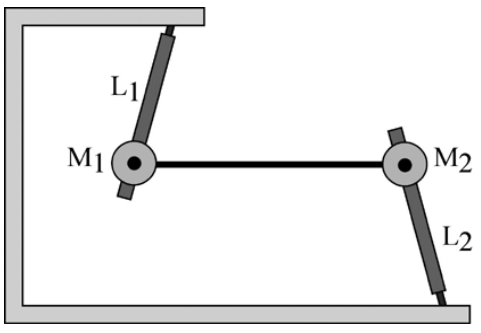

Fig. 1. Basic model of folded pendulum. A simple pendulum and an inverted pendulum are rigidly connected by a massless beam. The pendula arms are also massless, rigid, and provided with suitable flexures.

(STS-2 from Streckeisen AG), capable of 1.5-nm rms, would easily satisfy noise requirements, but they need onboard active electronics, with a nominal dissipated power of about $0.6 \mathrm{~W}$. To integrate them in the advanced LIGO vibration isolators a joint JILA-MIT-Stanford team have sealed the STS-2 inside suitable vacuum enclosures. To avoid this expense and complication, accelerometers with remote conditioning active electronics have been developed for VIRGO [4] and SAS systems. In this paper, we briefly present the main features of the new horizontal accelerometers designed for advanced SAS attenuators; they combine a noise level comparable with STS's, a completely passive in-vacuum readout, and superior performances in terms of cross-coupling between the sensitive axis and the other degrees of freedom. The last feature has been achieved by means of a monolithic folded pendulum (FP) mechanics which also ensures low resonant frequency, low Brownian noise, and compact size. The same design should also be used for new short-period seismometers, ultralow-noise cryogenic accelerometers, and modular sensors for seismic monitoring networks. These new perspectives in folded-pendulum-based instrumentation are discussed in the next sections.

\section{Monolithic Folded Pendulum}

In an FP the proof mass is suspended on one end by a simple pendulum and supported on the other by an inverted pendulum (IP). In this geometry the simple pendulum positive gravity restoring force is balanced by the IP negative one. The only dissipation is given by the flexures connecting the different parts of the system. The sketch of Fig. 1 shows the kinematics of the FP. In this model a simple pendulum, with mass $M_{1}$ and length $L_{1}$, and an inverted pendulum, with mass $M_{2}$ and length $L_{2}$, are connected by a massless rigid beam. The resonant frequency of the system is given by

$$
\omega_{0}=\sqrt{\left(\frac{M_{1}}{L_{1}}-\frac{M_{2}}{L_{2}}\right) \frac{g}{\left(M_{1}+M_{2}\right)}+\gamma}
$$




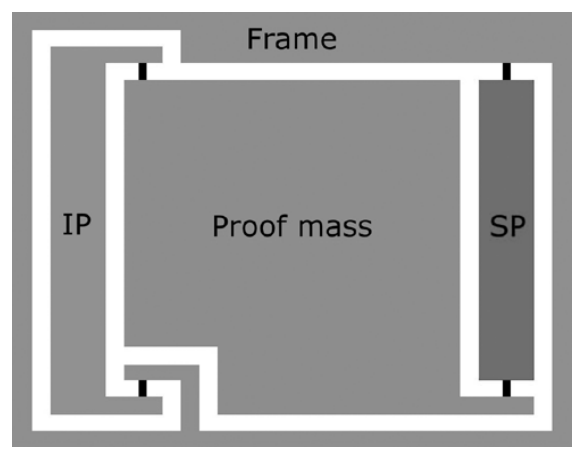

Fig. 2. Monolithic realization of a folded pendulum. The bottom left and top right flexures connect the inverted pendulum (IP) and the simple pendulum (SP) arms to the instrument frame. The proof mass is linked to the pendula by means of the top left and bottom right flexures.

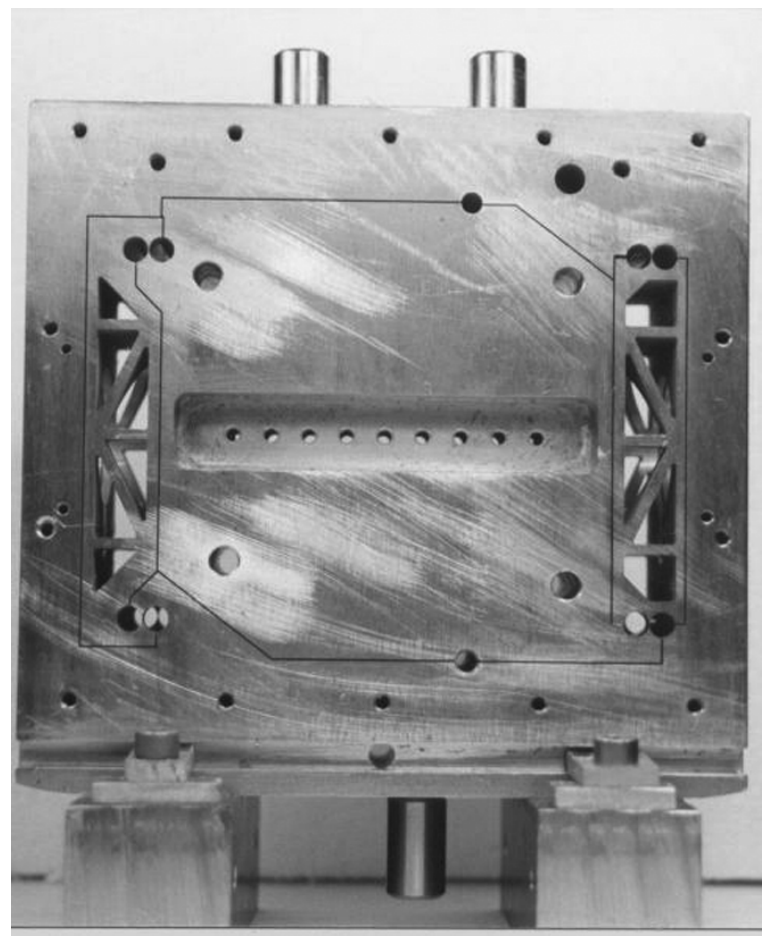

Fig. 3. Side view of the monolithic folded pendulum horizontal accelerometer we have designed for SAS advanced seismic attenuation systems. Two-second natural period is achieved by design.

where $\gamma$ represent the effect of cumulative flex-joint's stiffness. The resonant frequency can be lowered, in principle, arbitrarily by adjusting the center of mass position with respect to the two arms. Natural periods ranging from seconds to minutes can be achieved in compact sizes making the FP the best geometry for horizontal sensors. This configuration has been reproduced in a monolithic version (see Fig. 2) for the mechanics of the horizontal accelerometers of the advanced SAS seismic attenuation systems, by using a combination of traditional and wire electric discharge machining (EDM).

A side view of the monolithic FP accelerometer is proposed in Fig. 3. The mechanics is obtained from a $140 \times 134 \times 40 \mathrm{~mm}$ block of 7075 aluminum alloy. A thin $250-\mu$ m-wide EDM cut is used to separate the two pendulum arms and the proof mass from the frame; $50-\mu \mathrm{m}$-thick tensional circular notch flexures, four on each side of the accelerometer, connect the proof mass to the

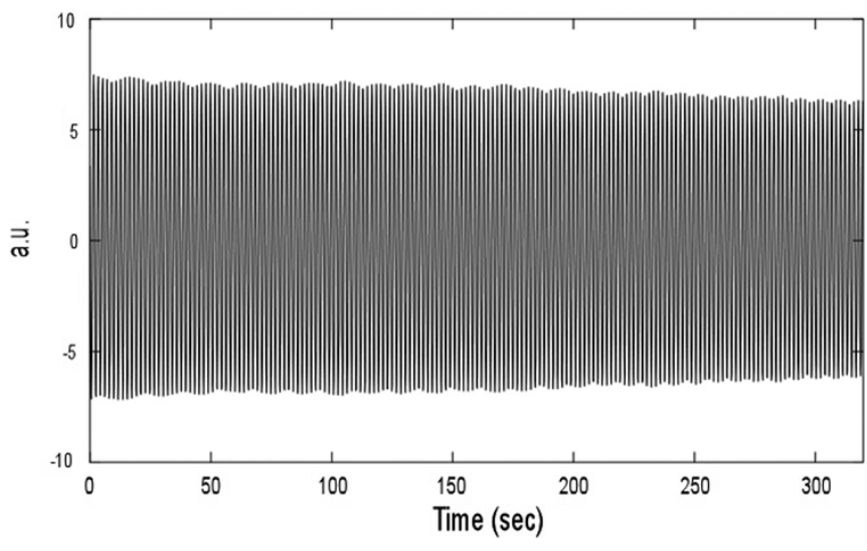

Fig. 4. Mechanical free decay measured in low vacuum. The natural period is $2 \mathrm{~s}$; the decay time of about $2000 \mathrm{~s}$ corresponding to $Q=3000$.

structure. The arms are $71.5 \mathrm{~mm}$ long, and the equivalent suspended mass is $0.83 \mathrm{~kg}$. The accelerometer was designed with the load almost exactly balanced on the two arms. In this way the instrument's behavior is dominated by the restoring force of the flexures; a resonant frequency of $540 \mathrm{mHz}$ has been achieved. The FP accelerometer normally operates under vacuum; hence, excluding gas damping, the only damping forces are localized in the flexures which allow the movement of the proof mass.

A $Q=3000$ mechanical quality factor is obtained at pressures below $10^{-2}$ mbar; a typical free decay time series is shown in Fig. 4. At atmospheric pressure the constrained air flow between the closely spaced surfaces of the EDM cut and the capacitive readout plates limits the quality factor to 7 .

In vacuum the Brownian motion amplitude of the proof mass emulates a frame inertial displacement of $0.5-\mathrm{nm}$ rms, which represents the ultimate resolution limit of the instrument.

The main feature of this mechanical design is the insensitivity to unwanted degrees of freedom. The entire machining, milling and EDM, are done with the full accuracy of numerical control machines; the equality of the two pendula length, their relative positioning and alignment, as well as the parallelism and equality of the eight flexures, are guaranteed within a few microns. This local and overall accuracy generates a cross-axis coupling of $0.01 \%$ that would be difficult to achieve in a composite structure. The details of the monolithic FP mechanics will be presented in [5].

\section{ForCE BALANCE ACCELEROMETER}

The instruments designed for the advanced SAS seismic isolation systems are configured as force-balance accelerometers: a capacitance position sensor detects the motion of the proofmass with respect to the frame; its output, properly filtered by a PID (proportional-integral-derivative) analog controller, drives a coaxial linear voice-coil actuator located at the opposite end of the mass (see the section view of the SAS accelerometer in Fig. 5).

The force actuator keeps dynamically the proof mass at the equilibrium position, corresponding to the capacitance sensor null point. The closed loop bandwidth is $150 \mathrm{~Hz}$, with $55^{\circ}$ of phase margin. Phase rotations are limited below $1^{\circ}$ up to $10 \mathrm{~Hz}$, as required for the application of the accelerometer as the sensor 


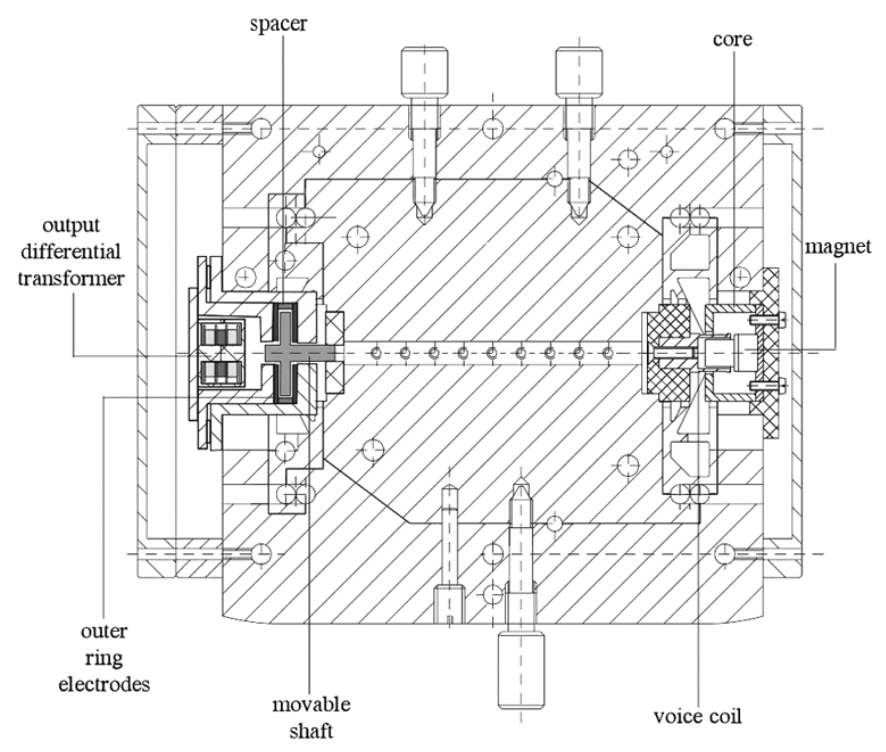

Fig. 5. Section view of the SAS force balance accelerometer. A differential capacitance position sensor, on the left, and a linear voice-coil actuator, on the right, are symmetrically located with respect to the proof mass.

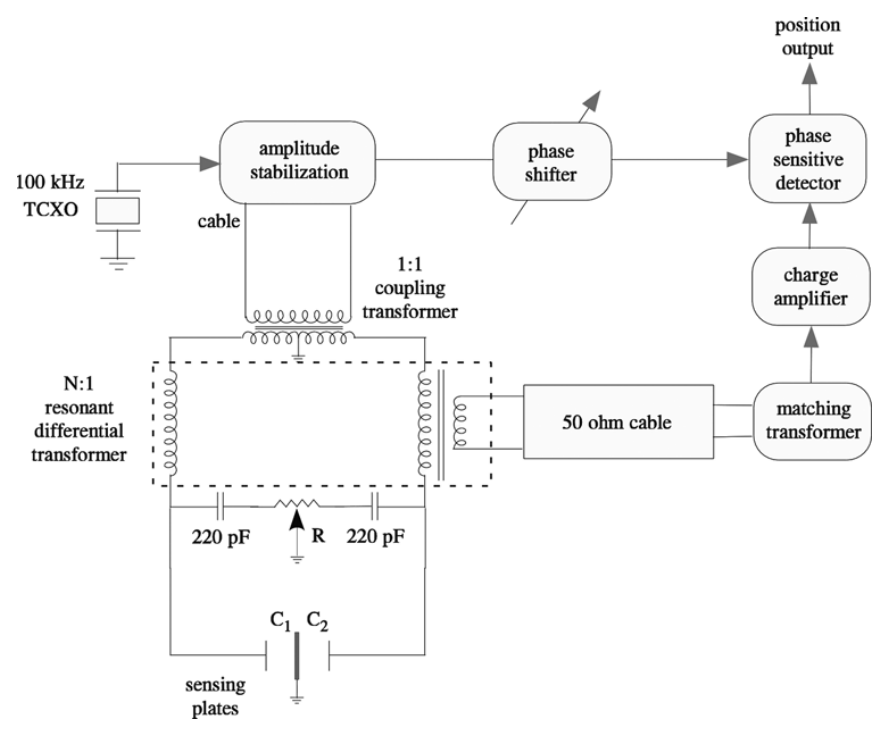

Fig. 6. Block scheme of the capacitance position sensor with remote signal conditioning.

in the inertial damping feedback loop. The sensor at the heart of the accelerometer is a three-plate differential capacitance sensor; the central electrode has the shape of a movable piston and is mounted on the test mass. Its motion is sensed by two coaxial ring electrodes separated by an insulating spacer and rigidly connected to the accelerometer frame. The capacitance bridge is coupled to the cablings and to the charge preamplifier by means of a custom-made resonant differential transformer (RDT) (see Fig. 6). Each primary winding of the RDT forms a series resonant circuit with the capacitance given by the sum of the sensing capacitor, stray capacitances and a fixed capacitor. In this configuration the total flux in the transformer is null when the piston is centered with respect to the reference rings.

Using a large step-down transformer ratio for the secondary winding, low impedance transmission of the signal was achieved and the effect of the cable capacitance was strongly

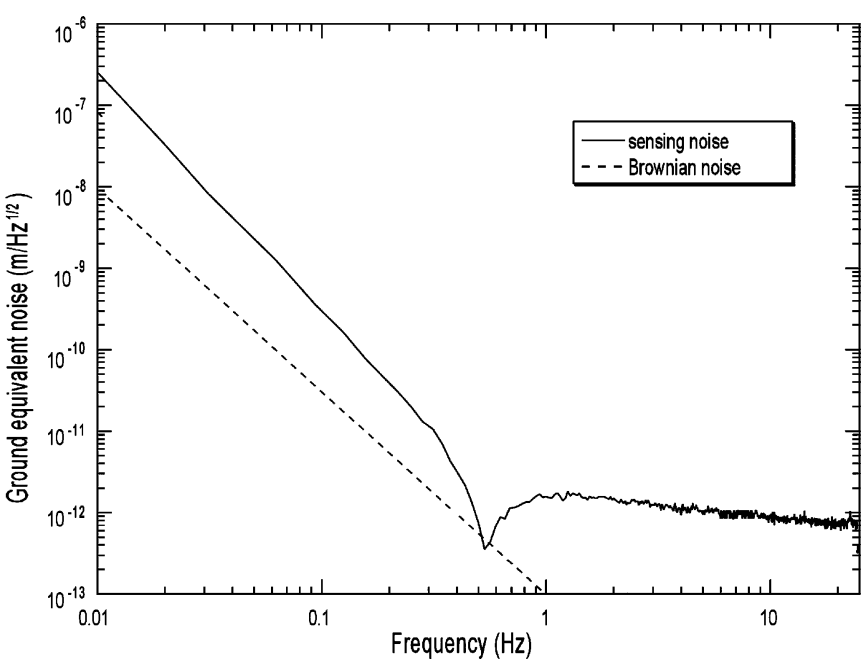

Fig. 7. Spectral density of the minimum detectable inertial displacement.

suppressed. In this way all active components were removed from the instrument and full high vacuum compatibility was achieved. No deterioration of the SNR have been observed with cables as long as $15 \mathrm{~m}$. The sensor is able to detect the position of the proof mass of the accelerometer with a noise level of 10 -pm rms integrated over all the frequencies from $0.01 \mathrm{~Hz}$.

In the same band the noise budget of this accelerometer, estimated from the contributions from Brownian noise, position readout noise and actuation noise, corresponds to an equivalent inertial displacement of 1-nm rms (see Fig. 7).

This result is comparable with STS-2 seismometer and exceeds the requirements for inertial damping applications. Many units of monolithic FP accelerometer will be operating in fall 2005 aboard the advanced seismic isolation systems of TAMA 300 gravitational wave detector.

\section{Folded Pendulum for Seismic Research}

Owing to compact size and high mechanical performances folded pendulum-based sensors look very promising for developing new geophysical instrumentation. A ruggedized design for on-field applications including vacuum sealing, an in-vacuum electromagnetic (or electrostatic) mass lock system for transit, as well as a remote recentering and calibration system is being studied. Besides the straightforward implementation of the FP accelerometer as short-period horizontal seismometer, new applications are being studied. Recently, a new compact tiltmeter for deep underground (boreholes) and ocean-bottom research has been proposed [6]. Seafloor tilt data have particular interest in earthquake research for tectonic dynamics studies and observation near subduction boundaries. The new tiltmeter is a rugged, modular instrument with full remote signal conditioning, suitable for array and mobile observation. A compact solid-state instrument is preferable with respect to classic large size water-tube tiltmeters and to electrolytic tiltmeters, affected by large long-term drifts. Horizontal pendula are directly sensitive to tilt motion; a tilt angle $\theta$ produces a displacement of the proof mass

$$
\Delta x=\frac{g}{\omega_{0}^{2}} \theta
$$




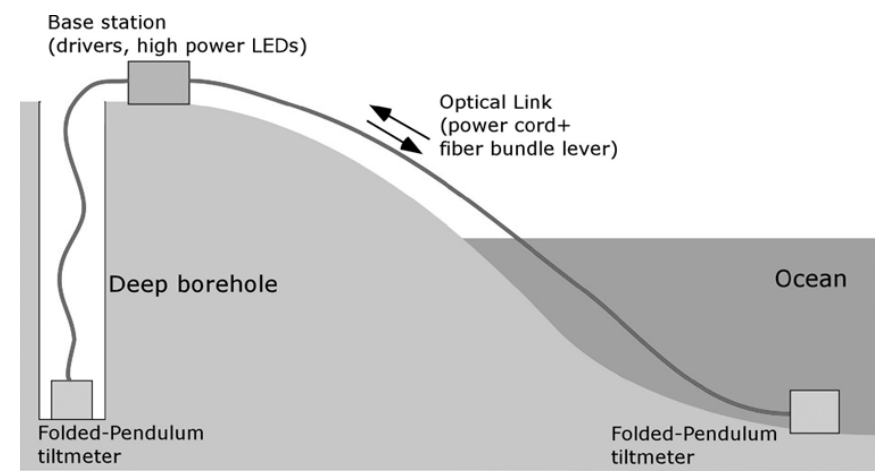

Fig. 8. Implementation of a deep borehole/sea-floor FP tiltmeter network. An optical link is used both to feed electrical power to the tiltmeter and to detect the position of its sensing mass.

with $g$ the Earth gravitational acceleration and $\omega_{0}$ the mechanical natural frequency. As an example, a 2-s period pendulum moves by $1 \mu \mathrm{m}$ for $1-\mu \mathrm{rad}$ tilt. In a folded pendulum, very long period of oscillation can be achieved resulting in high tilt amplification. The monolithic folded pendulum is also intrinsically low thermally sensitive; in the balanced load configuration only the resonant frequency is affected by temperature changes because of the thermoelastic coefficient of the flexure material ( $-300 \mathrm{ppm} /{ }^{\circ} \mathrm{C}$ for typical metal alloys). Tilt thermal drift are only possible because of mismatch in the length of the two pendula arms; the monolithic CNC machined mechanics allows to limit this effect below $10 \mathrm{nrad} /{ }^{\circ} \mathrm{C}$. Notice that for best electrolytic tiltmeters (http://www.geomechanics.com), the same parameter is $2 \mu \mathrm{rad} /{ }^{\circ} \mathrm{C}$. Applications in tight space (boreholes) and a hostile environment require a reduction of the size of the mechanical sensor. Building a folded pendulum in a few cubic centimeters volume without losing the low resonant frequency feature should be possible by changing the load distribution, making ultrathin flexures for hinges, or replacing the hinges with long monolithic membranes. A squeezing factor of $75 \%$ in volume, with respect to the TAMA FP accelerometer, is achievable by simply reducing the flexure thickness to $25 \mu \mathrm{m}$ by means of electrochemical machining. Alternative FP configuration are being evaluated to get further shrinking of the size.

For the folded pendulum monolithic tiltmeter a full remote signal conditioning will be implemented. The operating scheme is shown in Fig. 8.

The tiltmeter will be optically linked to the remote driver which could be, eventually, many kilometers apart. This kind of connection is bidirectional, robust, insensitive to electromagnetic noise, and chemically resistant. The fiber cord will deliver both the signal of the tiltmeter proof mass position and the electrical power for the servo control, yielding the instrument to be completely passive and virtually maintenance free. As well as for accelerometer, force balance operation is necessary for the damping of the pendulum natural frequency and to limit position sensor nonlinearities. The electrical power necessary for the force feedback actuator is generated by a high-power LED (1 W or more), transmitted through the optical link and converted to voltage by a silicon solar cell mounted aboard the tiltmeter. The position of the test mass of the tiltmeter is detected by a fiber optic bundle reflective sensor driven by an amplitude modulated LED.

This kind of optical lever has been demonstrated to be capable of nanometer accuracy over tens of microns of linear range [7]. Using a 2-s period FP mechanics, nanoradian accuracy will be achievable in tilt monitoring. We would also notice that for an eventual application of the FP accelerometers as modular seismic sensors, the use of CNC machining techniques would guarantee a repeatable performance specification, thereby lowering manufacturing costs from initial adjustment.

\section{CONCLUSION}

A review on new design monolithic folded pendulum accelerometers has been presented. The instruments are fully realized with numerically controlled machining for best alignment of the mechanical components. Four couples of widely spaced, parallel, flexures connecting the pendulum components allow strong reduction of spurious couplings between the sensitive axis and the other five degrees of freedom. Cross-axis residual sensitivity of $0.01 \%$ has been achieved. This feature makes the monolithic FP accelerometer suitable to be used in multiaxis active seismic isolation systems. These accelerometers, with an expected equivalent displacement noise of 1-nm rms integrated over all the frequencies from $0.01 \mathrm{~Hz}$, have been installed on TAMA 300 seismic attenuators in fall 2005.

Monolithic FP are also being studied for applications in geophysical instrumentation. Short-period seismometers and compact tiltmeters are being developed for deep borehole and seafloor seismic research.

\section{REFERENCES}

[1] E. Bougleux et al., "Seismic isolation by mechanical filters at very low frequencies," Nucl. Instrum. Meth., vol. A409, pp. 480-483, 1998.

[2] S. Marka et al., "Anatomy of TAMA SAS seismic attenuation system," Class. Quantum Grav., vol. 19, pp. 1605-1614, 2002.

[3] R. Abbott et al., "Seismic isolation for advanced LIGO," Class. Quantum Grav., vol. 19, pp. 1591-1597, 2002.

[4] S. Braccini et al., "Low noise wide-band accelerometer using an inductive displacement sensor," Rev. Sci. Instrum., vol. 66, pp. 2672-2676, 1995.

[5] A. Bertolini, R. DeSalvo, F. Fidecaro, M. Francesconi, S. Marka, V. Sannibale, D. Simonetti, A. Takamori, and H. Tariq, "Mechanical design of a single axis monolithic accelerometer for advanced seismic attenuation systems," Nucl. Instrum. Meth. A, 2006, to be published.

[6] A. Takamori, "Compact tiltmeter with folded pendulum," presented at the IRIS Broad-band Seismometer Workshop, Lake Tahoe, Mar. 24-26, 2004.

[7] A. Shimamoto and K. Tanaka, "Optical fiber bundle displacement sensor using an ac-modulated light source with subnanometer resolution and low thermal drift," Appl. Opt, vol. 34, pp. 5854-5860, 1995.

Author photographs and biographies not available at the time of publication. 